\title{
Multi-interacting dark matter in the Boltzmann code cLASS
}

\section{Deanna C. Hooper*}

Service de Physique Théorique, CP225,

Université Libre de Bruxelles, Boulevard du Triomphe, 1050 Bruxelles, Belgium

E-mail: deanna.hooper@ulb.be

Despite its remarkable success, the standard $\Lambda$ CDM paradigm has been challenged lately by significant tensions between different datasets. This has boosted interest in non-minimal dark sectors, which are theoretically well-motivated and inspire new search strategies for dark matter (DM). With this in mind, we have developed a new and efficient version of the Boltzmann code CLAss that allows for one DM species to have simultaneous interactions with photons, baryons, and dark radiation. As a proof-of-principle of this code, we have reassessed existing cosmological bounds on the various interaction coefficients in multi-interacting DM scenarios, showing that these constraints are robust to the underlying cosmological model. We have also investigated the ability of these models to alleviate the $H_{0}$ and $S_{8}$ tensions, showing that the combination of DM-photons and DM - dark radiation interactions can alleviate both tensions. The upcoming public release of our code will pave the way for the study of various rich dark sectors.

Tools for High Energy Physics and Cosmology - TOOLS2020

2-6 November, 2020

Institut de Physique des 2 Infinis (IP2I), Lyon, France

${ }^{*}$ Speaker 


\section{Introduction}

The cold dark matter (CDM) paradigm, which assumes cold and collisionless dark matter (DM) particles interacting only gravitationally, is a cornerstone of both cosmology and particle physics. However, despite the overwhelming success of CDM, and by extension of the standard $\Lambda \mathrm{CDM}$ cosmological model, increasingly more precise data have uncovered some tensions among different datasets.

On the one hand, the expansion rate of the universe (quantified with $H_{0}$ ) as inferred by CMB [1] measurements differs by more than $4.4 \sigma$ from that measured in the local universe [2-4], known as the Hubble tension. Moreover, the clustering of matter on scales of $\sim 8 \mathrm{Mpc} / \mathrm{h}$ (quantified with $S_{8}$ ) inferred from CMB data [1] is in more than $2 \sigma$ tension [5, 6] with the measurements obtained from weak lensing experiments [7-11] - this is known as the $S_{8}$ tension. Furthermore, there are possible shortcomings of CDM when looking at structure formation on small scales [12-19]. Combined with the EDGES anomaly [20,21] and the lack of detections in DM experiments, these tensions have called into question the CDM paradigm.

This has motivated interest in models beyond the standard CDM paradigm, such as Interacting Dark Matter (IDM), either with additional dark relativistic species (Dark Radiation, henceforth DR) or with Standard Model particles. Here and in [22] - which is the basis for this contribution - we aim, for the first time, to study scenarios in which the IDM has several simultaneous interactions, with three main objectives: first we will develop the formalism needed in order to describe these simultaneous interactions, which require non-trivial modifications, such as for the temperature evolution of the different species. Second, we will assess the cosmological bounds on different IDM cross sections in models with two or three simultaneous interactions, in order to check whether they differ from those obtained with single interactions, since in principle, some cancellations between the various effects could lead to parameter degeneracies. Third, we will study the possible implications these multi-interacting scenarios have on the aforementioned cosmological tensions.

In order to do this, we have developed a new version of the Boltzmann solver CLAss [23] featuring DM-DR interactions (already present since cLAss v2.9 [24, 25]), DM-baryon interactions, and DM-photon interactions in a unified and systematic approach, without substantial increase of the runtime. The code developed here will be made publicly available in a forthcoming release, CLASS v3.1.

\section{The Boltzmann code cLASS}

CLASS is designed to simulate the evolution of linear perturbations in the universe and to compute the cosmological observables for a given input model. CLAss was written by Julien Lesgourgues \& Thomas Tram, and first released in 2011 [23, 26], and aims at being

- General: it features numerous models and many different cosmological outputs.

- Modern: CLASs is written in C with a modular structure, and comes with a wrapper for both python and $\mathrm{C}++$. 
- User-friendly: it is well documented (both inside the code and with the comprehensive online documentation $^{1}$ ). The code is easy to follow and equations are not repeated, making it straightforward to modify. CLASs also comes with several example jupyter notebooks.

- Accurate and fast: agrees with самв [27] at the $10^{-4}(0.01 \%)$ level for CMB observables, and runs in a couple of seconds for most models.

- Up-to-date: continuously maintained and expanded with more models and features, while maintaining compatibility with older versions.

More information about CLASs, including previous courses and tutorials can be found on the CLASS home page ${ }^{2}$.

\section{Dark matter interactions}

We choose to describe the DM interactions at an effective level in the the form of temperaturedependent cross sections, rather than at a fundamental level in the form of Lagrangian densities. This means that we will treat different types of interactions as independent, even though they may be linked in a fundamental theory. We highlight that in this work and in the forthcoming CLAss v3.1 release, we only consider one single IDM species with potentially all of the relevant interaction channels.

\section{Dark matter - baryon interactions}

Scattering between DM and baryons can lead to an exchange of momentum proportional to the momentum transfer cross section

$$
\sigma_{\mathrm{T}}=\int \mathrm{d} \Omega \frac{\mathrm{d} \sigma}{\mathrm{d} \Omega}(1-\cos \theta) .
$$

In weakly-coupled theories, $\sigma_{\mathrm{T}}$ can only depend on even powers of the DM-baryon relative velocity $v$ and in many cases this dependence is given by a power law. In the present work we consider $\sigma_{\mathrm{T}}=\sigma_{\mathrm{DM}-b} v^{n_{b}}$ with $n_{b}=0$, which occus in contact interactions [28] (note, however, that cLAss can take any value of $\left.n_{b} \in[-4,4]\right)$.

We have implemented DM-baryon interactions in cLAss following the formalism described in Refs. [29-32], among others. Within this framework, it is assumed that both DM and baryons are non-relativistic (valid for DM masses above the $\mathrm{MeV}$ scale), and that in the early universe both species follow a Maxwell velocity distribution. With these assumptions, the DM Euler equation will gain an additional term

$$
\theta_{\mathrm{DM}}^{\prime}=\theta_{\mathrm{DM}, \mathrm{standard}}^{\prime}-\Gamma_{\mathrm{DM}-b}\left(\theta_{\mathrm{DM}}-\theta_{b}\right)
$$

where $\Gamma_{\mathrm{DM}-b}$ is the conformal DM-baryon momentum exchange rate, which will also appear in the modified baryon Boltzmann equations (and is called $R_{\chi}$ in e.g., Ref. [29]). Throughout this work,

\footnotetext{
${ }^{1}$ https://lesgourg.github.io/class_public/class_public-2.9.0/doc/manual/html/index.html
}

${ }^{2}$ http://www.class-code.net/ 
primes stand for derivatives with respect to conformal time, and conformal rates are defined with respect to conformal time. The rate $\Gamma_{\mathrm{DM}-b}$ is given by the deceleration of the DM bulk velocity. At leading order in the non-relativistic expansion, it reads

$$
\Gamma_{\mathrm{DM}-b}=\frac{a \rho_{b} \sigma_{\mathrm{DM}-b} c_{n_{b}}}{m_{\mathrm{DM}}+m_{b}}\left(\frac{T_{b}}{m_{b}}+\frac{T_{\mathrm{DM}}}{m_{\mathrm{DM}}}+\frac{V_{\mathrm{RMS}}^{2}}{3}\right)^{\frac{n_{b}+1}{2}} \mathcal{F}_{\mathrm{H} e},
$$

where $T_{x}$ and $m_{x}$ represent the temperature and mass of species $x$, and $\sigma_{\mathrm{DM}-b}$ is the DM-baryon cross section. In this work we focus on scattering only with hydrogen atoms, which requires setting the corrective factor $\mathcal{F}_{\mathrm{He}}$ to $1-Y_{p} \approx 0.76$ [31] and the average baryon mass $m_{b}$ to be equal to the proton mass $m_{p} \approx 0.938 \mathrm{GeV} / c^{2}$. Our approach can be generalised to include Helium scattering as in Ref. [29], while electron scattering is discussed below. The integration constant $c_{n_{b}}$ depends only on $n_{b}$ and for the case studied here is $c_{0}=2.1$. Finally, the velocity term in equation (3) is the averaged value of the DM bulk velocity relative to the baryon fluid, and is given by

$$
V_{\mathrm{RMS}}^{2} \equiv\left\langle V_{\mathrm{DM}}^{2}\right\rangle \simeq\left\{\begin{array}{ll}
10^{-8}, & z>10^{3} \\
10^{-8}\left(\frac{(1+z)}{10^{3}}\right)^{2}, & z \leq 10^{3}
\end{array} .\right.
$$

An important feature of these interactions is that they will substantially modify the baryon and DM temperature evolution, such that $T_{\mathrm{DM}}$ needs to be numerically evolved alongside $T_{b}$ and $x_{e}$. Since for $n_{b}>-3$, DM-baryon interactions couple the baryon and DM temperatures efficiently at early times ${ }^{3}$, we take $T_{\mathrm{DM}}=T_{b}$ as the initial condition. For $n_{b}<-3$, on the other hand, the interactions are negligible at early times, for which we would take an initial temperature $T_{\mathrm{DM}} \simeq 0$.

Finally, we note in passing that models with DM-baryon interactions would typically also feature DM-electron interactions. However, the cross section for the latter is expected to be suppressed proportional to $\mu_{\chi e}^{2} / \mu_{\chi p}^{2}$, where $\mu$ denotes the reduced mass. In certain models, for example if the interactions arise from the exchange of a scalar mediator, even stronger suppression is possible. Therefore, we do not consider these interactions here, even though they would be straightforward to implement in the formalism presented above, as explained in Ref. [32].

\section{Dark matter - photon interactions}

Here we focus on the case in which DM-photon interactions are independent of temperature and result in an additional term in the DM and photon velocity equations, analogous to the standard baryon-photon interaction term. The Euler equation for DM will thus be modified as

$$
\theta_{\mathrm{DM}}^{\prime}=\theta_{\mathrm{DM}, \text { standard }}^{\prime}-\Gamma_{\mathrm{DM}-\gamma}\left(\theta_{\mathrm{DM}}-\theta_{\gamma}\right)
$$

where

$$
\Gamma_{\mathrm{DM}-\gamma}=\frac{4 \rho_{\gamma}}{3 \rho_{\mathrm{DM}}} a \sigma_{\mathrm{DM}-\gamma} n_{\mathrm{DR}}
$$

\footnotetext{
${ }^{3}$ To quickly assess whether a given rate is efficient on cosmological time scales, one should compare it to the conformal Hubble rate $\mathcal{H}=a^{\prime} / a$, related to the usual Hubble rate by $\mathcal{H}=a H$.
} 
is the conformal DM-photon momentum exchange rate, $\sigma_{\mathrm{DM}-\gamma}$ is the DM-photon elastic scattering cross section, and $n_{\mathrm{DR}}=\rho_{\mathrm{DM}} / m_{\mathrm{DM}}$ is the DM number density.

Following Refs. [33, 34], it is convenient to define the scattering cross section relative to the Thompson cross section $\sigma_{\mathrm{Th}}$, and to introduce the dimensionless parameter

$$
u_{\mathrm{DM}-\gamma}=\frac{\sigma_{\mathrm{DM}-\gamma}}{\sigma_{\mathrm{Th}}}\left(\frac{m_{\mathrm{DM}}}{100 \mathrm{GeV}}\right)^{-1},
$$

such that

$$
\sigma_{\mathrm{DM}-\gamma}=6.65 \times 10^{-29} u_{\mathrm{DM}-\gamma}\left(\frac{m_{\mathrm{DM}}}{100 \mathrm{GeV}}\right) \mathrm{m}^{2} .
$$

\section{Dark matter - dark radiation interactions}

In analogy with the DM-photon interactions discussed above, DM can also interact with other forms of radiation. We consider the possibility that DM interacts with massless relics from the dark sector, called generically DR, which have negligible interactions with Standard Model particles. The general framework for such interactions has been developed in the ETHOS formalism [35], which also describes in detail the mapping between the underlying particle physics model and its effects on structure formation observables. The ETHOS parametrisation assumes that a single DM species interacts with a relativistic component via the 2-to-2 scattering DM $+\mathrm{DR} \leftrightarrow \mathrm{DM}+\mathrm{DR}$. In addition we also include DR self-interactions via the process DR $+\mathrm{DR} \leftrightarrow \mathrm{DR}+\mathrm{DR}$, following the ETHOS implementation in CLAss from Refs. [24, 25]. We further assume that DR maintains a thermal spectrum with $T_{\mathrm{DR}} \propto(1+z)$ and vanishing chemical potential (such that $n_{\mathrm{DR}} \propto T_{\mathrm{DR}}^{3}$ ) throughout the times relevant for CMB physics and until today. Finally, the Euler equation for DM gains an additional term:

$$
\theta_{\mathrm{DM}}^{\prime}=\theta_{\mathrm{DM}, \text { standard }}^{\prime}-\Gamma_{\mathrm{DM}-\mathrm{DR}}\left(\theta_{\mathrm{DM}}-\theta_{\mathrm{DR}}\right),
$$

where $\Gamma_{\mathrm{DM}-\mathrm{DR}}$ is the conformal DM-DR momentum exchange rate. The DR perturbations are described by a Boltzmann hierarchy integrated over momentum, like in the case of massless neutrinos. When the DR self-interactions are assumed to be very strong, we truncate these equations at the level of the first two multipoles, like for a relativistic perfect fluid.

We consider the case in which the interaction rate appearing in the DR equations has a powerlaw dependence on temperature and can thus be written as

$$
\Gamma_{\mathrm{DR}-\mathrm{DM}}=\omega_{\mathrm{DM}} a_{\mathrm{dark}}\left(\frac{1+z}{1+z_{d}}\right)^{n_{\mathrm{DR}}},
$$

where $\omega_{\mathrm{DM}}=\Omega_{\mathrm{DM}, 0} h^{2}$, while the rate $a_{\mathrm{dark}}$ gives the overall interaction strength close to $z_{d}, n_{\mathrm{DR}}$ is the power-law dependence of the temperature, and $1+z_{d}$ is a normalisation factor. The scattering rate for $\mathrm{DM}$ is given by

$$
\Gamma_{\mathrm{DM}-\mathrm{DR}}=\left(\frac{4}{3} \frac{\rho_{\mathrm{DR}}}{\rho_{\mathrm{DM}}}\right) \Gamma_{\mathrm{DR}-\mathrm{DM}},
$$

which is proportional to $(1+z)^{n_{\mathrm{DR}}+1}$, due to the different redshift dependence of $\rho_{\mathrm{DR}}$ and $\rho_{\mathrm{DM}}$. In principle, one can also calculate the self-scattering rate $\Gamma_{\mathrm{DR}-\mathrm{DR}}$ for a given model, but in the case 
of strong self-coupling, DR behaves like a perfect fluid and the precise value of $\Gamma_{\mathrm{DR}-\mathrm{DR}}$ becomes irrelevant.

Once the pivot redshift $z_{d}$ of equation (10) has been fixed arbitarily to $z_{d}=10^{7}$, like in previous works [24, 25, 35], the conformal DM-DR momentum exchange rate (10) can be conveniently parametrised either in terms of $\left(a_{\mathrm{dark}} a_{0}^{-1}\right)$ or of the current rate

$$
\Gamma_{\mathrm{DM}-\mathrm{DR}}^{0} \equiv \Gamma_{\mathrm{DM}-\mathrm{DR}}(z=0) a_{0}^{-1}=\frac{4}{3} \omega_{\mathrm{DR}} a_{\mathrm{dark}} a_{0}^{-1} 10^{-7 n_{\mathrm{DR}}},
$$

with $\omega_{\mathrm{DR}}=\Omega_{\mathrm{DR}, 0} h^{2}$, such that

$$
\Gamma_{\mathrm{DM}-\mathrm{DR}}=\frac{4}{3} \omega_{\mathrm{DR}} a_{\mathrm{dark}}(1+z)\left(\frac{1+z}{10^{7}}\right)^{n_{\mathrm{DR}}}=\Gamma_{\mathrm{DM}-\mathrm{DR}}^{0} a_{0}(1+z)^{1+n_{\mathrm{DR}}}
$$

In the case of $n_{\mathrm{DR}}=\{2,4\}$, Refs. [24, 25] report their observational bounds on the parameter $a_{\text {dark }}$ (assuming $a_{0}=1$ and $z_{d}=10^{7}$ ). For $n_{\mathrm{DR}}=0$, Refs. [25, 36, 37] report bounds on $\Gamma_{\mathrm{DM}-\mathrm{DR}}^{0}$, which is just denoted by $\Gamma_{0}$ in these works.

Here we focus on a model known to be particularly relevant for the discussion of the Hubble and $S_{8}$ tensions $[25,36,37]$. In this model, one chooses $n_{\mathrm{DR}}=0$ such that $\Gamma_{\mathrm{DM}-\mathrm{DR}} / \mathcal{H}$ remains constant throughout radiation domination, and decreases during matter domination. Then, the small but cumulative effect of DM-DR scattering throughout radiation domination can lead to a small enhancement of DR fluctuations and to a small suppression of DM fluctuations.further assumes that DM-DR interactions are too weak to bring the two species into thermal equilibrium, while DR has strong self-interactions and behaves as a perfect fluid (not free-streaming). This class of models is easy to motivate with a concrete dark sector set-up, like for instance in the non-Abellian Dark Matter model of Ref. [38]. It can be described by two parameters

$$
\left(\Gamma_{\mathrm{DM}-\mathrm{DR}}^{0}, \Delta N_{\mathrm{DR}}\right),
$$

where $\Delta N_{\mathrm{DR}} \equiv \frac{\rho_{\mathrm{DR}}}{\rho_{1 v}}$ gives the amount of DR relative to the energy density of a single neutrino species in the instantaneous decoupling approximation. We emphasize that for a given model of $\mathrm{DR}$, this parameter also fixes the DR temperature $T_{\mathrm{DR}}$. We consider the case that DR has two bosonic degrees of freedom, which implies

$$
\Delta N_{\mathrm{DR}} \approx 8.8 \times\left(\frac{T_{\mathrm{DR}}}{T_{\gamma}}\right)^{4} .
$$

For the parameters that we will consider, the DR does not thermalise with either photons or DM and, therefore, $\Delta N_{\mathrm{DR}}$ (or equivalently $T_{\mathrm{DR}}$ ) is a free parameter.

\section{Cosmological effects of multi-interacting dark matter}

The impact of each single DM scattering channel has been described in several previous works. Using our multi-interaction code, we find empirically that these effects tend to sum up in a rather straightforward manner, such that the effects of dual or triple interactions are very similar to the 

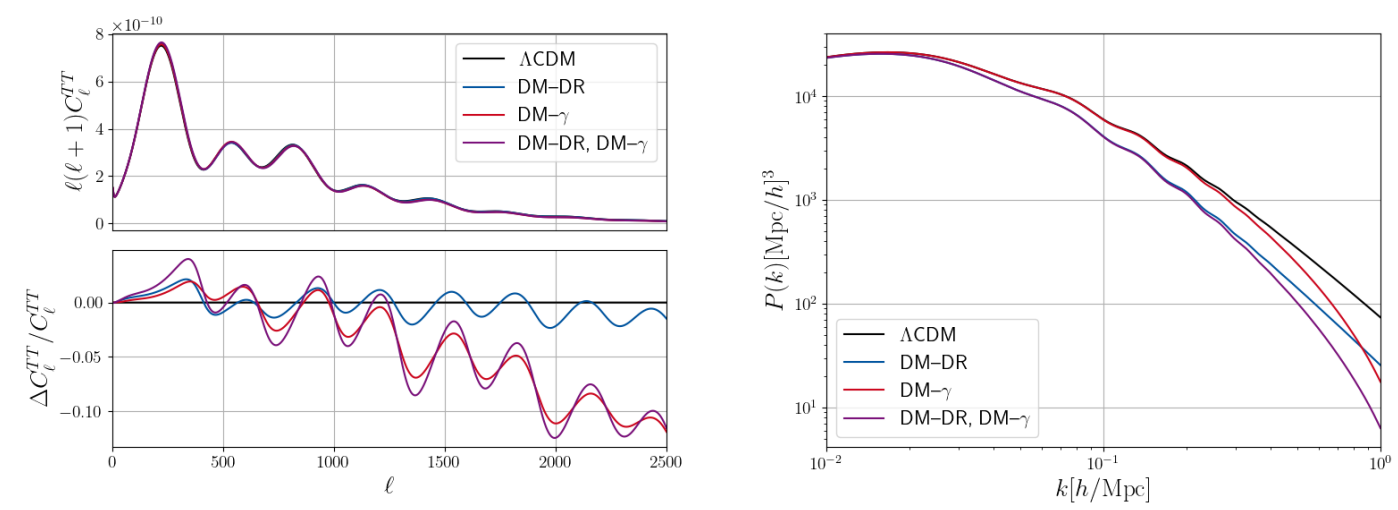

Figure 1: Left: Effect of (individual or combined) DM-DR and DM-photon interactions on the CMB temperature anisotropy spectra, with relative residuals. Right: Effect of (individual or combined) DM-DR and DM-photon interactions on the matter power spectrum.

summed effects from each channel. This can be seen at the level of the CMB and matter power spectra for individual models, illustrated in figure 1 for the particular example of DM interacting simultaneously with photons and DR $\left(n_{\mathrm{DR}}=0\right)$. For these figures, we have assumed $m_{\mathrm{DM}}=1 \mathrm{GeV}$, $N_{\mathrm{DR}}=0.07, \Gamma_{\mathrm{DM}-\mathrm{DR}}^{0}=5 \times 10^{-7} \mathrm{Mpc}^{-1}$, and $u_{\mathrm{DM}-\gamma}=10^{-3}$.

The DM-photon interactions have the following effects: suppress the small-scale CMB spectra due to collisional damping, shift the peaks to smaller scales due to a reduction of the sound speed, and suppress the small-scale matter power spectrum exponentially due to the DM being dragged by the photons $[33,34,39]$. These effects are clearly visible when comparing the black and red curves in figure 1. The DM-DR interactions with $n_{\mathrm{DR}}=0$ have a smaller effect on the CMB. Normally, extra free-streaming radiation suppresses the small-scale CMB spectrum due to Silk damping and shifts the acoustic peaks due to neutrino drag. These effects are much smaller with the DR component of the $n_{\mathrm{DR}}=0$ model, because small-scale photon perturbations are also boosted by the DR perturbations, which are larger than those of free-streaming neutrinos due to the DM-DR scattering. Furthermore, the $n_{\mathrm{DR}}=0$ model suppresses the matter power spectrum in a special way, due to DM being dragged by DR over the radiation dominated epoch. The suppression is smoother and affects larger scales than with other interacting DM models [36, 37]. These effects can also be seen in the blue curves in figure 1. Finally, in all panels, one can check that the combined effect of simultaneous DM-DR and DM-photon interactions (purple curves) looks qualitatively very similar to the sum of the individual effects, showing that these effects are largely additive.

\section{Dark matter interactions in CLASS}

\section{Input parameters in CLASS}

As is standard practice in CLASs, each new species is identified by a short acronym, which allows for a quick search of all of the relevant equations. As we are considering only one IDM species with multiple interactions, the relevant species are interacting dark matter (idm) and interacting dark radiation (idr). Additionally, to find the specifics of each type of interaction for the IDM, the 


\begin{tabular}{|l|c|c|}
\hline This work & CLASS v3.1 & CLASS v2.9 [25] \\
\hline$m_{\mathrm{DM}}$ & m_idm & - \\
\hline$\sigma_{\mathrm{DM}-b}$ & cross_idm_b & - \\
$n_{b}$ & n_index_idm_b & - \\
\hline$\sigma_{\mathrm{DM}-\gamma}$ & cross_idm_g & - \\
$u_{\mathrm{DM}-\gamma}$ & u_idm_g & - \\
\hline$a_{\text {dark }}$ & a_idm_dr & a_dark \\
$\Gamma_{\text {DM-DR }}^{0}$ & Gamma_0_idm_dr & Gamma_0_nadm \\
$N_{\mathrm{DR}}$ & N_idr & N_dg \\
$\xi$ & xi_idr & xi_idr \\
$n_{\text {DR }}$ & n_index_idm_dr & nindex_dark \\
\hline
\end{tabular}

Table 1: Correspondence between the notation of this work and the input parameters for CLASs v3.1 and CLASS v2.9 (for DM-DR interactions).

following acronyms are employed: idm_b, for DM-baryon interactions, idm_g, for DM-photon interactions, and idm_dr, for DM-DR interactions. The relevant input parameters used in CLASS for the different IDM models are summarised in table 1.

\section{Temperature evolution of multi-interacting dark matter}

In the combined multi-interacting DM model, the DM temperature needs to be calculated consistently and evolved together with the baryon temperature. The value of the DM temperature is relevant for the calculation of the DM-baryon momentum exchange rate given by equation (3), and for that of the DM sound speed appearing in the DM Euler equation. The evolution equation for the DM temperature depends on all interaction rates

$$
\begin{aligned}
T_{\mathrm{DM}}^{\prime}=-2 \mathcal{H} T_{\mathrm{DM}} & -2 \Gamma_{\mathrm{DM}-\gamma}\left(T_{\mathrm{DM}}-T_{\gamma}\right) \\
& -2 \Gamma_{\mathrm{DM}-\mathrm{DR}}\left(T_{\mathrm{DM}}-T_{\mathrm{DR}}\right) \\
& -\frac{2 m_{\mathrm{DM}}}{m_{\mathrm{DM}}+m_{b}} \Gamma_{\mathrm{DM}-b}\left(T_{\mathrm{DM}}-T_{b}\right) .
\end{aligned}
$$

The rates in front of each term $\left(T_{\mathrm{DM}}-T_{x}\right)$ are the conformal heat exchange rates between DM and each species $x$. They are related to the respective momentum exchange rates, because they are derived from the same collision operator in the Boltzmann equation. Assuming that each scatter changes the momentum of the non-relativistic DM particle only by a small amount, one can analytically derive $\Gamma_{\mathrm{DM}-x}^{\text {heat }}=2 \Gamma_{\mathrm{DM}-x}^{\text {momentum }}$ for scattering with a massless species, and a similar relation with additional mass factors for non-relativistic scattering partners (such as baryons).

To follow the temperature evolution of equation (16), we need to impose initial conditions for the DM temperature at the earliest time considered by the cLAss thermodynamics module. By default, this time would correspond to the redshift $z_{\text {ini }}=5 \cdot 10^{6}$, but in the presence of IDM the cLAss thermodynamics module starts earlier. We consider two main scenarios, described below. 
First, in the models where there is a strong coupling at early times (such as DM-photon, DM-baryon with $n_{b}>-3$, or DM-DR with $n_{\mathrm{DR}}>0$ ) we use an analytic approximation of the decoupling redshifts to find the latest decoupling redshift $z_{\mathrm{dec}}$. This is then used to determine the starting point of integration, which is taken to be $z_{\mathrm{ini}}=10^{4} z_{\mathrm{dec}}$. At these times the coupling is strong enough to justify tightly coupled initial conditions, and we can set $T_{\mathrm{DM}}=T_{b}=T_{\gamma}=T_{\gamma}^{0}(1+z)$ for DM-photon and DM-baryon couplings, and $T_{\mathrm{DM}}=T_{\mathrm{DR}}=T_{\mathrm{DR}}^{0}(1+z)$ for DM-DR interactions.

Otherwise, in those cases where there is no strong coupling at early times, the starting redshift of integration is set to $z_{\text {ini }}=10^{8}$. If there are DM-DR interactions with $n_{\mathrm{DR}}=0$, we can use the steady-state attractor solution of $T_{\mathrm{DM}}=\frac{\epsilon}{(1+\epsilon)} T_{\mathrm{DR}}$, where $\epsilon \equiv 2 \Gamma_{\mathrm{DM}-\mathrm{DR}} / \mathcal{H}$ is constant throughout radiation domination. Finally, in the case of only DM-baryon interactions with $n_{b}=-4$, we assume that DM was either never in thermal equilibrium with photons at high temperatures or became much colder than photons due to several entropy releases after DM decoupling, and following previous authors (see e.g. Ref. [32]), cLAss will start from a null value of $T_{\mathrm{DM}}$ at $z_{\mathrm{ini}}=10^{8}$.

Following the DM temperature $T_{\mathrm{DM}}(z)$ is mainly useful for getting a correct estimate of the DM-baryon momentum exchange rate following equation (3) even in the presence of other interactions such as DM-photons or DM-DR. This is important for computing CMB observables and matter power spectra, since it impacts the evolution of the matter and baryon density fluctuations $\left(\delta_{\mathrm{DM}}, \delta_{b}\right)$. It is also important for following the evolution of $T_{b}(z)$, and thus potentially for using observations of the IGM temperature and ionization fraction, of the $21 \mathrm{~cm}$ differential brightness temperature, of the Sunyaev-Zeldovitch effect, or of CMB spectral distortions.

\section{Tight-coupling approximations with multi-interacting dark matter}

The models described here feature multiple possible combinations of tight-coupling regimes between photons, baryons, DM, and DR. Whenever two or more species are tightly coupled, the system of perturbation equations becomes stiff. Fortunately, cLASs is using by default an implict ODE solver, ndf15, which is ideal for solving stiff systems [23]. Such an ingredient is crucial in the context of this work, because otherwise we would need to implement a complicated set of Tight-Coupling Approximations (TCAs) describing fifteen possible tight-coupling regimes between two, three, or four species. However, there is a limit to the degree of stiffness that ndf15 can handle. Thus, when a scattering rate exceeds the Hubble rate by many orders of magnitude, it is still advisable to switch from the exact equations to TCA equations, which are derived from a perturbative expansion of the solution of the equation for the differential velocity $\left(\theta_{x}-\theta_{y}\right)$ in the inverse scattering rate $[23,40]$.

\section{Code Performance}

In table 2 we show the average runtime of the code for the different interacting models, using the $2 \sigma$ limits from table 3 for the interaction rates, $m_{\mathrm{DM}}=1 \mathrm{GeV}$ and $N_{\mathrm{DR}}=0.5$ (if DM-DR interactions are active). All runtime checks were performed on 8 CPUs on a Dell XPS with Intel Core i7-8665U CPU $(1.90 \mathrm{GHz})$. It is worth pointing out that none of the interacting models considered in this work cause a significant slowdown of the code, at most slowing it down by $\sim 70 \%$, and remaining always under $1.5 \mathrm{~s}$ runtime. 


\begin{tabular}{|l|c|c|}
\hline Case & Runtime [s] & \% Slowdown \\
\hline$\Lambda \mathrm{CDM}$ & 0.936 & 0.0 \\
\hline $\mathrm{DM}-\mathrm{b}$ & 0.950 & 1.5 \\
$\mathrm{DM}-\gamma$ & 0.940 & 0.4 \\
$\mathrm{DM}-\mathrm{DR}, n_{\mathrm{DR}}=0$, fluid DR & 1.307 & 39.6 \\
$\mathrm{DM}-\mathrm{DR}, n_{\mathrm{DR}}=0$, free-streaming DR & 2.181 & 132.9 \\
\hline $\mathrm{DM}-\mathrm{b}+\mathrm{DM}-\gamma$ & 0.983 & 4.9 \\
$\mathrm{DM}-\mathrm{b}+\mathrm{DM}-\mathrm{DR}, n_{\mathrm{DR}}=0$, fluid DR & 1.340 & 43.1 \\
$\mathrm{DM}-\gamma+\mathrm{DM}-\mathrm{DR}$ & 1.356 & 44.8 \\
\hline $\mathrm{DM}-\mathrm{b}+\mathrm{DM}-\gamma+\mathrm{DM}-\mathrm{DR}, n_{\mathrm{DR}}=0$, fluid DR & 1.396 & 49.1 \\
\hline
\end{tabular}

Table 2: Average performance for a subset of the DM interaction models considered here.

\section{Cosmological constraints}

To constrain the different scattering rates involved in multi-interacting DM models we will run MCMC scans using the parameter extraction code MontePython [41, 42]. All of our parameter scans will also allow the $\Lambda \mathrm{CDM}$ parameters to vary freely with flat priors. We use the Planck 2018 baseline dataset [43] including temperature, polarisation and CMB lensing. Additionally, we include a BAO data, using measurements of $D_{V} / r_{\text {drag }}$ by 6dFGS at $z=0.106$ [44], by SDSS from the MGS galaxy sample at $z=0.15$ [45], and additionally by BOSS from the CMASS and LOWZ galaxy samples of SDSS-III DR12 at $z=0.2-0.75$ [46]. We also include new data from the DR14 eBOSS release, namely QSO clustering at $z=1.52$ [47], BAO measurements from Lyman- $\alpha$ forest autocorrelation at $z=2.34$ [48], and from cross correlation of Lyman- $\alpha$ and QSO [49] at $z=2.35$. We refer to these datasets henceforth simply as BAO.

First we will test the individual interaction models, in order to compare our results to previous studies. We will focus on the case of $m_{\mathrm{DM}}=1 \mathrm{GeV}$, and constrain the parameters $\left\{\sigma_{\mathrm{DM}-b}, u_{\mathrm{DM}-\gamma}, N_{\mathrm{DM}}\right.$ and $\left.\Gamma_{\mathrm{DM}-\mathrm{DR}}^{0}\right\}$ for DM-baryon, DM-photon, and DM-DR interactions respectively. We will take flat priors on all parameters except for $\Gamma_{\mathrm{DM}-\mathrm{DR}}^{0}$, where we impose $\Gamma_{\mathrm{DM}-\mathrm{DR}}^{0}<10^{-7}$ to avoid a bi-modality in the posterior distribution. The resulting $2 \sigma$ upper bounds $(95.4 \% \mathrm{CL})$ for all interactions considered are shown in table 3.

For the case of DM-baryon interactions, we compare to Ref. [31], where our bound on $\sigma_{\mathrm{DM}-b}$ improves by a factor $\sim 1.5$, which can be attributed to the improvement obtained when using Planck 18 instead of Planck 15, and a more complete set of BAO data. For the case of DM-photon interactions, our bound is looser than the result obtained in Ref. [34] based on Planck 15 (TTTEEE + lowTEB + lensing) data by about $20 \%$. This shift is likely due to the difference in the inferred optical depth of reionization between these datasets. For the case of DM-DR interactions, our results are relatively close to the most recent bounds on this model taken from Ref. [25], in spite of the different choice of prior and of the updated CMB and BAO data set. However, a positive correlation between $N_{\mathrm{DM}}$ and $\Gamma_{\mathrm{DM}-\mathrm{DR}}^{0}$ does not appear anymore: in presence of non-zero DM-DR interactions, the bounds on the DR abundance can only get stronger. This is because the more accu- 


\begin{tabular}{|l|c|c|c|c|c|}
\hline $\begin{array}{l}\text { Parameter } \\
\text { Units }\end{array}$ & $\begin{array}{c}\sigma_{\mathrm{DM}-b} \\
{\left[10^{-25} \mathrm{~cm}^{2}\right]}\end{array}$ & $\begin{array}{c}u_{\mathrm{DM}-\gamma} \\
{\left[10^{-4}\right]}\end{array}$ & $\begin{array}{c}\Gamma_{\mathrm{DM}-\mathrm{DR}}^{0} \\
{\left[10^{-8}\right]}\end{array}$ & $\begin{array}{c}H_{0} \\
{[\mathrm{~km} / \mathrm{s} / \mathrm{Mpc}]}\end{array}$ & $S_{8}$ \\
\hline \hline$\Lambda \mathrm{CDM}$ & - & - & - & $67.70 \pm 0.43$ & $0.825 \pm 0.011$ \\
\hline $\mathrm{DM}-\mathrm{b}$ & 2.2 & - & - & $67.70 \pm 0.43$ & $0.813 \pm 0.014$ \\
$\mathrm{DM}-\gamma$ & - & 1.8 & - & $67.70 \pm 0.43$ & $0.803 \pm 0.021$ \\
$\mathrm{DM}-\mathrm{DR}$ & - & - & 6.2 & $68.73 \pm 0.96$ & $0.813 \pm 0.014$ \\
\hline $\mathrm{DM}-\mathrm{b}+\mathrm{DM}-\gamma$ & 2.3 & 1.7 & - & $67.70 \pm 0.44$ & $0.793 \pm 0.021$ \\
$\mathrm{DM}-\mathrm{b}+\mathrm{DM}-\mathrm{DR}$ & 1.9 & - & 6.7 & $68.66 \pm 0.93$ & $0.810 \pm 0.014$ \\
$\mathrm{DM}-\gamma+\mathrm{DM}-\mathrm{DR}$ & - & 1.6 & 5.5 & $68.75 \pm 0.94$ & $0.799 \pm 0.020$ \\
\hline $\mathrm{DM}-\mathrm{b}+\mathrm{DM}-\gamma+\mathrm{DM}-\mathrm{DR}$ & 1.9 & 1.5 & 6.1 & $68.62 \pm 0.90$ & $0.791 \pm 0.019$ \\
\hline
\end{tabular}

Table 3: Summary of the $2 \sigma$ upper bounds $(95.4 \% \mathrm{CL})$ on the different interaction parameters for all of the $\mathrm{DM}$ interaction models considered here, assuming a mass of $m_{\mathrm{DM}}=1 \mathrm{GeV}$. Summary of the mean and $1 \sigma$ (68\% CL) bounds on $H_{0}$ and $S_{8}$ for all of the DM interaction models considered here

rate measurement of the high- $\ell$ CMB polarisation spectrum allows to better discriminate between the Silk damping effect induced by a higher $N_{\mathrm{DM}}$ and the gravitational boost effect induced by a higher $\Gamma_{\mathrm{DM}-\mathrm{DR}}^{0}$, and thus, by more clustered DR.

Since our code allows several interactions to be switched on simultaneously, we can address for the first time the question of possible degeneracies between the different interaction channels. In principle, effects from individual interactions could cancel each other, open degeneracy directions in parameter space, and allow to relax some of the bounds. Thus, to some extent, we are probing here the model dependence of CMB bounds on DM interactions.

In figure 2 we show the joint 2D confidence contours on each pair of momentum exchange rate parameters when two interactions are turned on (in the foreground), and when three interactions are turned on (in the background) for all of the models considered here. All of the resulting $2 \sigma$ upper bounds are also shown in table 3 , which allows for quick comparison of the bounds in the different interacting scenarios. The resulting contours are shaped like triangles or quarters-of-an-ellipse, suggesting that a larger interaction of one type typically requires a smaller interaction of the other type, and showing no degeneracies between the parameters. This in turn implies that the various effects are additive and only their sum is constrained, and none of the individual bounds (which correspond to the edge of the contours when one of the two parameters is zero) can be relaxed by the combined effects. This implies that the bounds are robust to the underlying cosmological model (at least for the models considered here).

We now wish to assess the ability of each model to address the $H_{0}$ and $S_{8}$. In the last two columns of table 3, we also show the marginalised confidence intervals for the Hubble parameter $H_{0}$ and the clustering amplitude parameter $S_{8}$. For reference, we also show in the first column the $\Lambda \mathrm{CDM}$ values, obtained using the same pipeline and datasets. We can check that the preferred range for $H_{0}$ is in $4.3 \sigma$ tension with the late-time measurement of Ref. [3], $H_{0}=74.03 \pm 1.42 \mathrm{~km} / \mathrm{s} / \mathrm{Mpc}$, while the preferred range for $S_{8}$ is in $2.3 \sigma$ tension with the conservative results of Ref. [10], 


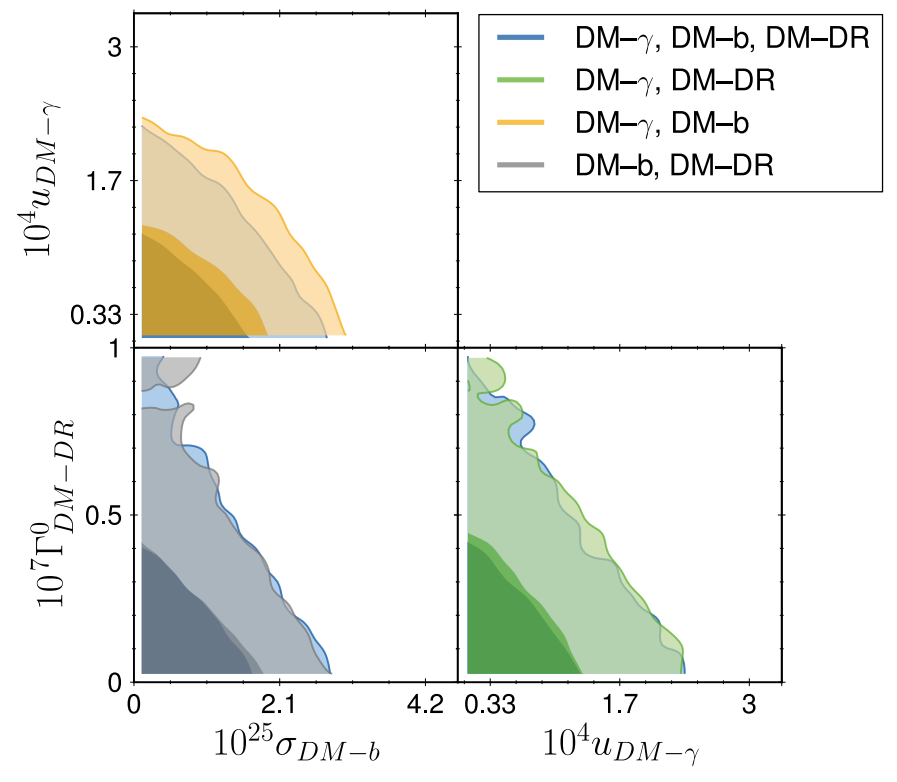

Figure 2: $68.3 \% \mathrm{CL}$ and $95.4 \% \mathrm{CL}$ contours of the momentum exchange rate parameters for the various interactions with $n_{b}=0$.

$S_{8}=0.762 \pm 0.025$

Por the case of DM-baryon interactions, the predictions for $H_{0}$ are unaffected, as this model does not incorporate any mechanism to counteract an increase in $H_{0}$. On the other hand, the value of $S_{8}$ is significantly affected. In this case, CMB bounds are compatible with values of the momentum exchange rate that lead to a suppression of the matter power spectrum on scales that are relevant for $S_{8}$. Note that the inclusion of Lyman- $\alpha$ data would result in stronger bounds on the momentum exchange rate [31], which would restrict the possibility to lower $S_{8}$.

The DM-photon interactions can efficiently reduce $S_{8}$ : the matter power spectrum is suppressed on small scales because DM density fluctuations remain as small as photon fluctuations as long as the two species are coupled. As already discussed in Ref. [34], the CMB puts bounds on $u_{\mathrm{DM}-\gamma}$ that are compatible with a reduction of the matter power spectrum on scales relevant for $S_{8}$. We find that this is still the case with our Planck $18+$ BAO dataset: the $S_{8}$ tension gets reduced from the $2.3 \sigma$ to the $1.3 \sigma$ level by the DM-photon interaction. We should, however, keep in mind that our comment on the DM-baryon case applies also to this case: the reduction of $S_{8}$ might become marginal if we used Lyman- $\alpha$ data to put stronger bounds on $u_{\mathrm{DM}-\gamma}$.

For DM-DR interactions, we confirm the findings of Refs. [25, 36, 37] in that the DM-DR interaction model with $n_{\mathrm{DR}}=0$ can reduce both tensions by a moderate amount (from $4.3 \sigma$ to $3.1 \sigma$ for $H_{0}$, and from $2.3 \sigma$ to $1.8 \sigma$ for $S_{8}$ ). The increase in $H_{0}$ is mainly due to the presence of self-interacting DR, and the decrease in $S_{8}$ is due to the drag effect of DR on DM. However, as mentioned before, with the inclusion of Planck 2018 data the model can no longer alleviate the $H_{0}$ tension substantially. 

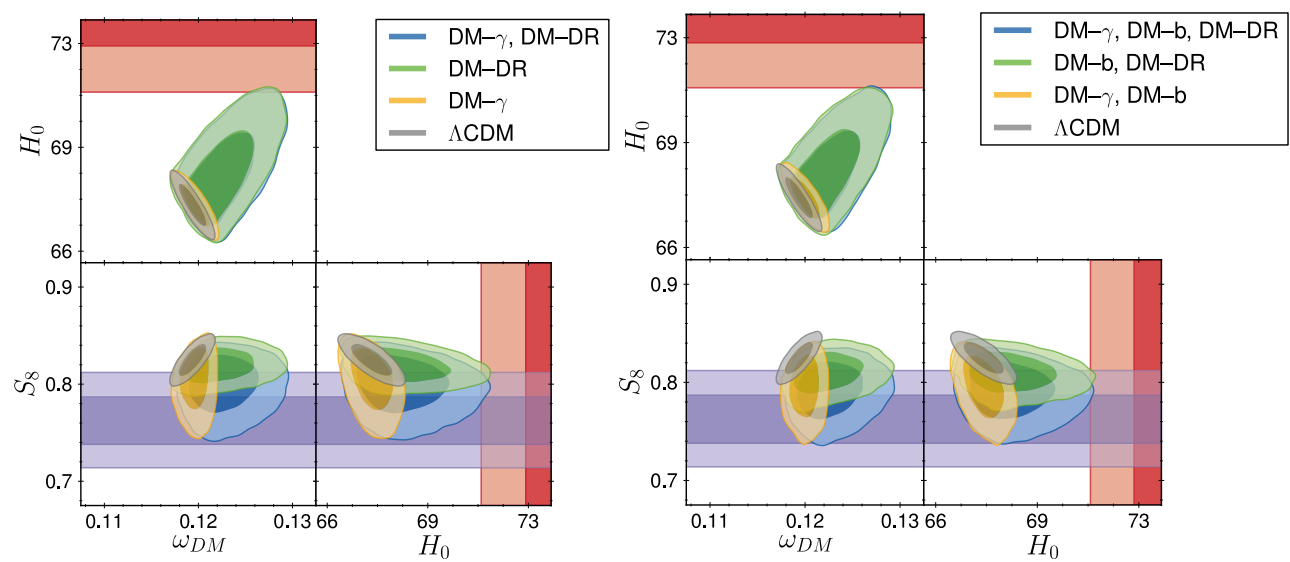

Figure 3: $68.3 \% \mathrm{CL}$ and $95.4 \% \mathrm{CL}$ contours of $\left(H_{0}, S_{8}, \omega_{\mathrm{DM}}\right)$, assuming various interactions with different temperature dependencies. We show for comparison the case of the the $\Lambda$ CDM model, as well as the $S_{8}$ measurement of [10] in purple and the $H_{0}$ determination of [3] in red. Left: Single interactions with DR and photons, as well as the corresponding double interaction. Right: Double interactions with baryons and photons or DR, as well as the triple interaction case.

Since DM-photon interactions offer the most efficient way to reduce $S_{8}$, and DM-DR to increase $H_{0}$, we check the effects of the combined model for the cosmological tensions. The results are shown in the line labelled DM- $\gamma+$ DM-DR in table 3, and are well summarised by the left panel of figure 3. In this case, the tensions get simultaneously reduced from $4.3 \sigma$ to $3.1 \sigma$ for $H_{0}$, and from $2.3 \sigma$ to $1.2 \sigma$ for $S_{8}$. The figure shows very clearly that the confidence contours of the combined model incorporate a large region of parameter space with high $H_{0}$ and low $S_{8}$ which would be incompatible with the data in each single interaction model. Of course, we should keep in mind that this is done at the expense of introducing three new parameters. Finally, the right panel of figure 3 confirms that switching on the DM-baryon interactions with $n_{b}=0$ on top of the other two channels has no further impact on the cosmological tensions.

\section{Discussion}

Motivated by a series of unexplained tensions in cosmological data, we have developed a new version of CLAss which allows for the DM to have multiple simultaneous interaction channels. Our code features DM-baryon, DM-photon, and DM-DR interactions (this last one already present in CLASs v2.9 [24, 25]), and allows these interaction channels of the DM species to be switched on simultaneously without making the Boltzmann code significantly slower. This code will constitute the version 3.1 of CLASS.

As a proof-of-principle of our code, we have used it to investigate the cosmological effects of multiple DM interactions. Our first main result, summarised in figure 2, is that when multiple interactions are switched on, there are no counteracting effects leading to parameter degeneracies and to a relaxation of CMB bounds on individual momentum exchange rates. Our second result, demonstrated by figure 3 , is that the combination of several interaction channels can help to reduce 
the cosmological tensions. Specifically, if we have simultaneous DM-photon and DM-DR interactions, we can alleviate both the $H_{0}$ and the $S_{8}$ tensions, but at the expense of introducing three extra free parameters with respect to the minimal $\Lambda \mathrm{CDM}$ model.

The upcoming public release of our code will pave the way towards the study of more complicated dark sector models, in which there could be multiple DM relics, decays within the dark sector, effects of inelastic scattering, or transitions between energy levels if the dark sector contains dark atoms. Studies of such extended dark sectors may bring more convincing explanations of the $H_{0}$ and $S_{8}$ tensions, and potentially of other unexplained observations such as the EDGES anomaly or the small scale crisis.

\section{Acknowledgments}

DH is supported by the FNRS research grant number F.4520.19. Simulations were performed with computing resources granted by RWTH Aachen University under project jara0184 and thes 0811 .

\section{References}

[1] Planck collaboration, Y. Akrami et al., Planck 2018 results. I. Overview and the cosmological legacy of Planck, 1807.06205.

[2] L. Knox and M. Millea, Hubble constant hunter's guide, Phys. Rev. D 101 (2020) 043533, [1908.03663].

[3] A. G. Riess, S. Casertano, W. Yuan, L. M. Macri and D. Scolnic, Large Magellanic Cloud Cepheid Standards Provide a 1Determination of the Hubble Constant and Stronger Evidence

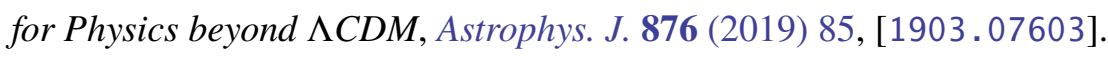

[4] L. Verde, T. Treu and A. Riess, Tensions between the Early and the Late Universe, 7, 2019. 1907. 10625. DOI.

[5] N. MacCrann, J. Zuntz, S. Bridle, B. Jain and M. R. Becker, Cosmic Discordance: Are Planck CMB and CFHTLenS weak lensing measurements out of tune?, Mon. Not. Roy. Astron. Soc. 451 (2015) 2877-2888, [1408.4742].

[6] LSST Dark Energy Science collaboration, C. Chang et al., A Unified Analysis of Four Cosmic Shear Surveys, 1808.07335.

[7] C. Heymans et al., CFHTLenS tomographic weak lensing cosmological parameter constraints: Mitigating the impact of intrinsic galaxy alignments, Mon. Not. Roy. Astron. Soc. 432 (2013) 2433, [1303. 1808].

[8] M. Asgari et al., KiDS+VIKING-450 and DES-Y1 combined: Mitigating baryon feedback uncertainty with COSEBIs, Astron. Astrophys. 634 (2020) A127, [1910. 05336].

[9] DES collaboration, T. M. C. Abbott et al., Dark Energy Survey Year 1 Results: Cosmological Constraints from Cluster Abundances and Weak Lensing, 2002 . 11124. 
[10] S. Joudaki et al., KiDS+VIKING-450 and DES-YI combined: Cosmology with cosmic shear, 1906.09262.

[11] C. Heymans, T. Tröster, M. Asgari, C. Blake, H. Hildebrandt, B. Joachimi et al., KiDS-1000 Cosmology: Multi-probe weak gravitational lensing and spectroscopic galaxy clustering constraints, arXiv e-prints (July, 2020) arXiv:2007.15632, [2007.15632].

[12] R. A. Flores and J. R. Primack, Observational and theoretical constraints on singular dark matter halos, Astrophys. J. Lett. 427 (1994) L1-4, [astro-ph/9402004].

[13] B. Moore, Evidence against dissipationless dark matter from observations of galaxy haloes, Nature 370 (1994) 629.

[14] A. A. Klypin, A. V. Kravtsov, O. Valenzuela and F. Prada, Where are the missing Galactic satellites?, Astrophys. J. 522 (1999) 82-92, [astro-ph/9901240].

[15] W. de Blok, The Core-Cusp Problem, Adv. Astron. 2010 (2010) 789293, [0910. 3538].

[16] M. Boylan-Kolchin, J. S. Bullock and M. Kaplinghat, Too big to fail? The puzzling darkness of massive Milky Way subhaloes, Mon. Not. Roy. Astron. Soc. 415 (2011) L40, [1103. 0007].

[17] M. Boylan-Kolchin, J. S. Bullock and M. Kaplinghat, The Milky Way's bright satellites as an apparent failure of LCDM, Mon. Not. Roy. Astron. Soc. 422 (2012) 1203-1218, [1111.2048].

[18] K. A. Oman et al., The unexpected diversity of dwarf galaxy rotation curves, Mon. Not. Roy. Astron. Soc. 452 (2015) 3650-3665, [1504 . 01437].

[19] S. Tulin and H.-B. Yu, Dark Matter Self-interactions and Small Scale Structure, Phys. Rept. 730 (2018) 1-57, [1705. 02358].

[20] J. D. Bowman, A. E. E. Rogers, R. A. Monsalve, T. J. Mozdzen and N. Mahesh, An absorption profile centred at 78 megahertz in the sky-averaged spectrum, http://arxiv.org/abs/1810.05912v1.

[21] R. Barkana, Possible interaction between baryons and dark-matter particles revealed by the first stars, Nature 555 (2018) 71-74, [1803.06698].

[22] N. Becker, D. C. Hooper, F. Kahlhoefer, J. Lesgourgues and N. Schöneberg, Cosmological constraints on multi-interacting dark matter, 2010.04074.

[23] D. Blas, J. Lesgourgues and T. Tram, The Cosmic Linear Anisotropy Solving System (CLASS) II: Approximation schemes, JCAP 07 (2011) 034, [1104 . 2933].

[24] M. Archidiacono, S. Bohr, S. Hannestad, J. H. Jørgensen and J. Lesgourgues, Linear scale bounds on dark matter-dark radiation interactions and connection with the small scale crisis of cold dark matter, JCAP 1711 (2017) 010, [1706.06870]. 
[25] M. Archidiacono, D. C. Hooper, R. Murgia, S. Bohr, J. Lesgourgues and M. Viel, Constraining Dark Matter-Dark Radiation interactions with $C M B, B A O$, and Lyman- $\alpha$, JCAP 10 (2019) 055, [1907.01496].

[26] J. Lesgourgues, The Cosmic Linear Anisotropy Solving System (CLASS) I: Overview, 1104.2932.

[27] A. Lewis, A. Challinor and A. Lasenby, Efficient computation of CMB anisotropies in closed FRW models, Astrophys. J. 538 (2000) 473-476, [astro-ph/9911177].

[28] X.-1. Chen, S. Hannestad and R. J. Scherrer, Cosmic microwave background and large scale structure limits on the interaction between dark matter and baryons, Phys. Rev. D65 (2002) 123515, [astro-ph/0202496].

[29] C. Dvorkin, K. Blum and M. Kamionkowski, Constraining Dark Matter-Baryon Scattering with Linear Cosmology, Phys. Rev. D89 (2014) 023519, [1311 . 2937].

[30] J. B. Muñoz, E. D. Kovetz and Y. Ali-Haïmoud, Heating of Baryons due to Scattering with Dark Matter During the Dark Ages, Phys. Rev. D92 (2015) 083528, [1509. 00029].

[31] W. L. Xu, C. Dvorkin and A. Chael, Probing sub-GeV Dark Matter-Baryon Scattering with Cosmological Observables, Phys. Rev. D97 (2018) 103530, [1802 . 06788].

[32] T. R. Slatyer and C.-L. Wu, Early-Universe constraints on dark matter-baryon scattering and their implications for a global $21 \mathrm{~cm}$ signal, Phys. Rev. D98 (2018) 023013, [1803.09734].

[33] R. J. Wilkinson, J. Lesgourgues and C. Bœhm, Using the CMB angular power spectrum to study Dark Matter-photon interactions, JCAP 1404 (2014) 026, [1309. 7588].

[34] J. Stadler and C. Bohm, Constraints on $\gamma$-CDM interactions matching the Planck data precision, JCAP 1810 (2018) 009, [1802 . 06589].

[35] F.-Y. Cyr-Racine, K. Sigurdson, J. Zavala, T. Bringmann, M. Vogelsberger and C. Pfrommer, ETHOS - an effective theory of structure formation: From dark particle physics to the matter distribution of the Universe, Phys. Rev. D 93 (2016) 123527, [1512 . 05344].

[36] J. Lesgourgues, G. Marques-Tavares and M. Schmaltz, Evidence for dark matter interactions in cosmological precision data?, JCAP 1602 (2016) 037, [1507.04351].

[37] M. A. Buen-Abad, M. Schmaltz, J. Lesgourgues and T. Brinckmann, Interacting Dark Sector and Precision Cosmology, JCAP 1801 (2018) 008, [1708.09406].

[38] M. A. Buen-Abad, G. Marques-Tavares and M. Schmaltz, Non-Abelian dark matter and dark radiation, Phys. Rev. D92 (2015) 023531, [1505 . 03542].

[39] C. Bœhm and R. Schaeffer, Constraints on dark matter interactions from structure formation: Damping lengths, Astron. Astrophys. 438 (2005) 419-442, [astro-ph/0410591]. 
[40] C.-P. Ma and E. Bertschinger, Cosmological perturbation theory in the synchronous and conformal Newtonian gauges, Astrophys. J. 455 (1995) 7-25, [astro-ph/9506072].

[41] B. Audren, J. Lesgourgues, K. Benabed and S. Prunet, Conservative Constraints on Early Cosmology: an illustration of the Monte Python cosmological parameter inference code, JCAP 02 (2013) 001, [1210.7183].

[42] T. Brinckmann and J. Lesgourgues, MontePython 3: boosted MCMC sampler and other features, Phys. Dark Univ. 24 (2019) 100260, [1804.07261].

[43] Planck collaboration, N. Aghanim et al., Planck 2018 results. VI. Cosmological parameters, 1807.06209.

[44] F. Beutler, C. Blake, M. Colless, D. H. Jones, L. Staveley-Smith, L. Campbell et al., The $6 d F$ Galaxy Survey: Baryon Acoustic Oscillations and the Local Hubble Constant, Mon. Not. Roy. Astron. Soc. 416 (2011) 3017-3032, [1106 . 3366].

[45] A. J. Ross, L. Samushia, C. Howlett, W. J. Percival, A. Burden and M. Manera, The clustering of the SDSS DR7 main Galaxy sample? I. A 4 per cent distance measure at $z=0.15$, Mon. Not. Roy. Astron. Soc. 449 (2015) 835-847, [1409. 3242].

[46] BOSS collaboration, S. Alam et al., The clustering of galaxies in the completed SDSS-III Baryon Oscillation Spectroscopic Survey: cosmological analysis of the DR12 galaxy sample, Mon. Not. Roy. Astron. Soc. 470 (2017) 2617-2652, [1607. 03155].

[47] M. Ata et al., The clustering of the SDSS-IV extended Baryon Oscillation Spectroscopic Survey DR14 quasar sample: first measurement of baryon acoustic oscillations between redshift 0.8 and 2.2, Mon. Not. Roy. Astron. Soc. 473 (2018) 4773-4794, [1705 . 06373].

[48] V. de Sainte Agathe et al., Baryon acoustic oscillations at $z=2.34$ from the correlations of Ly $\alpha$ absorption in eBOSS DR14, Astron. Astrophys. 629 (2019) A85, [1904 . 03400].

[49] M. Blomqvist et al., Baryon acoustic oscillations from the cross-correlation of Ly $\alpha$ absorption and quasars in eBOSS DR14, Astron. Astrophys. 629 (2019) A86, [1904.03430]. 